\title{
Factors Influencing Prescription Drug Synchronization: The Complex Role of Number of Medications
}

\author{
Joan M. Neuner, MD, MPH; Nicole Fergestrom, MS; Purushottam W. Laud, PhD; and Liliana Pezzin, PhD
}

\begin{abstract}
BACKGROUND: Despite the well-documented association of medication refill synchronization with medication adherence, little is known about how best to measure synchronization at pharmacy visits or about its relationship to number of medications.

OBJECTIVE: To examine the relationship of a commonly cited synchronization measure with the number of prescription medications.

METHODS: Using a cohort of women aged 66-90 years with stage 0-3 hormone receptor-positive breast cancer from the Surveillance, Epidemiology and End Result (SEER)-Medicare data, we identified women with pharmacy claims for at least 1 endocrine therapy prescription and at least 1 other medication fill. Twelve-month medication refill synchronization was calculated as the quotient of the number of pharmacy visits and the number of filled medications subtracted from 1. Multiple linear regression (including polynomials) was then used to assess the relationship between refill synchronization, number of medications, and other potentially influential factors.
\end{abstract}

RESULTS: Over $47 \%$ of cohort subjects took more than 10 unique medications. Subjects made an average (SD) of 29.9 (18.0) pharmacy visits, resulting in a mean (SD) synchronization of $0.28(0.18$, range $=0.0-0.92)$. The number of medications, including powers through to the fourth, was strongly associated with refill synchronization, with a rapid initial rise followed by a gradual increase after 10 medications. Although patient age and race/ethnicity were not associated with synchronization, there was a significant positive association of receipt of a low-income subsidy and residence in rural areas with synchronization.

CONCLUSIONS: There is a complex relationship between refill synchronization and number of prescribed medications, and future research into synchronization should account for this.

J Manag Care Spec Pharm. 2019;25(6):714-18

Copyright @ 2019, Academy of Managed Care Pharmacy. All rights reserved.

\section{What is already known about this subject}

Medication synchronization has been associated with medication adherence in studies that also adjust for number of medications. A strongly positive correlation between number of medications and synchronization has been noted.

\section{What this study adds}

Among a cohort of Medicare Part D enrollees newly initiating oral treatments for breast cancer, synchronization and the number of medications were found to have a complex, nonlinear relationship that should be accounted for in future studies.

Residing in less metropolitan areas and receipt of a low-income subsidy for prescription drugs were also associated with higher synchronization.
$\mathrm{M}$ edication synchronization, defined as minimizing the number of pharmacy visits needed to obtain medication fills, appears to foster medication adherence. Previous research has shown an association between synchronization and adherence to prescribed medicines for hypertension, hyperlipidemia, and several other chronic conditions. ${ }^{1,2}$ In one of the largest studies, adherence among hyperlipidemia patients with the lowest overall refill synchronization was $8 \%$ lower than among those with the highest synchronization. ${ }^{1}$ Several independent pharmacy groups and at least 1 pharmacy chain have now begun to implement programs to improve synchronization. ${ }^{3-5}$ Patients who enroll in such programs appear to have better adherence, ${ }^{3-5}$ and synchronization programs are now estimated to be available to up to 1 in 8 U.S. patients. ${ }^{6}$

Despite the growing attention to improving synchronization, few studies have provided detail about how best to measure it. The longest-established measure defines synchronization by subtracting from 1 the quotient of the number of visits and the number of medications filled. ${ }^{1}$ While appealing for its simplicity, this measure has important limitations, including a positive correlation with the subject's number of medications, ${ }^{1}$ which has not been fully explored. Furthermore, little is known about risk factors associated with poorer synchronization. Interventions to improve synchronization can require a substantial investment in pharmacy staff time, ${ }^{3}$ and many previous studies have mostly been limited to select populations.

Optimal measurement of synchronization is thus important to health delivery and health policy questions such as the effectiveness, cost-effectiveness, sustainability, and equitable access of synchronization programs. We explored this gap in the literature by examining a commonly cited synchronization measure in a retrospective study with a particular focus on its correlation with number of patient prescription medications. As part of a larger study into barriers to the use of oral cancer drugs, we used a cohort of women initiating a cancer medication, and we also examined sociodemographic and diseaserelated factors that we hypothesized might also influence synchronization, using a retrospective cohort.

\section{Methods}

\section{Data Sources and Study Population}

The main data source for this study was the populationbased Surveillance, Epidemiology, and End Results (SEER)Medicare dataset of Medicare beneficiaries with cancer. ${ }^{7}$ 
Detailed sociodemographic information was available from SEER, along with information on medical care, including prescription medications, from linked Medicare claims files and county-level information from census data. ${ }^{8}$

The study population of interest consisted of women aged 66-89 years with an incident, stage 0-3 hormone positive breast cancer in 2012 who were enrolled in Medicare Part A and Part B (to allow ascertainment of comorbidity) and in a Medicare Part D stand-alone prescription drug plan. Subjects who received an initial prescription for adjuvant endocrine therapy with tamoxifen or an aromatase inhibitor (anastrozole, exemestane, or letrozole) within 1 year after diagnosis $(\mathrm{n}=5,573)$ were included in the study. These once-daily oral medications are self-administered at home, are recommended for 5-10 years to prevent recurrence, ${ }^{9}$ and have coverage mandated by all Medicare Part D plans.

All medications were identified by generic name from the Medicare drug file, with confirmation using the unique medication identifiers from Medi-Span software (Wolters Kluwers, Hudson, $\mathrm{OH}$ ) so that unique medications could each be counted once. To allow ascertainment of all oral prescription medications during a 1-year study period, subjects were further required to continue enrollment in a Medicare Part D prescription drug plan $(n=5,528)$ and remain alive throughout the 12-month period after initial endocrine therapy prescription $(n=5,359)$. Given the focus on overall refill synchronization and its predictors, subjects were also required to have complete data on predictors $(n=5,295)$ and to have filled at least 1 other medication prescription during the first study months ( $\mathrm{n}=5,248)$.

This study was approved by the Medical College of Wisconsin Institutional Review Board and a waiver of informed consent was granted.

\section{Measuring Refill Synchronization}

To calculate synchronization of prescription fills, we used the synchronization measure developed by Choudhry et al. (2011). ${ }^{1}$ We defined synchronization over 12 months as 1 -(number of pharmacy visit dates $\div$ number of unique medication prescriptions).

\section{Independent Variables}

Women's age; race (white, black/African American, Asian, or other); ethnicity (Hispanic or non-Hispanic); enrollment in the Medicare Part D prescription drug program for low-income beneficiaries; and urban/rural residence were ascertained from the SEER-Medicare linked files. Comorbidity was calculated for the 1 year before breast cancer surgery using the breast cancer-specific National Cancer Institute algorithm for outpatient and inpatient diagnoses in Medicare files, ${ }^{10}$ which was adapted to allow use of outpatient claims in addition to the inpatient records used by Charlson and Deyo, ${ }^{11-13}$ and was categorized as 0,1 , or $\geq 2$. Other breast cancer treatments (radiation therapy, cytotoxic, ${ }^{14}$ or targeted chemotherapy ${ }^{15}$ ) were assessed from Medicare claims as in previous studies.

\section{Statistical Analysis}

Baseline demographics were described with means and proportions in summary statistics. Linear regression models were used to determine the association between independent variables and synchronization during the 12 months after the first endocrine therapy prescription. Because others have reported a correlation between the number of medications and synchronization (but the nature of that association has not been well described), we examined synchronization as a polynomial function of number of medications. Informed by the results, we then examined the association of number of medications in a model that also adjusted for patient race, prescription insurance status, and all other variables shown in Table 1. Because examination of marginal effects of covariates suggested categories could be combined, rural/urban was collapsed into 4 categories (Table 1), and comorbidity scores were shown as 0 or $\geq 1$. A linear regression model was used with adjustment for all variables included as listed in Table 1 . We repeated all of these analyses using only maintenance medications as defined by Medi-Span, which are mostly commonly used, long-term medications for chronic conditions. We examined the number of these medications as the independent variable and the synchronization of them as the dependent variable among the slightly smaller cohort $(n=5,126)$ who had at least 2 of these medications.

Given the inherent difficulty in interpreting the underlying coefficients from the polynomial relationship, we also calculated model-predicted synchronization in our all-medication cohort to illustrate the effect size of characteristics that had the largest correlation with synchronization. The predicted synchronization was computed at the individual level by setting the relevant variable to new values, while holding all other factors constant at their original (i.e., each individual's actual characteristics) levels. Model-predicted outcomes were calculated for each subject in the sample and then averaged across the total population.

\section{Results}

The study cohort consisted of 5,248 women with a mean (standard deviation [SD]) age of 74.4 (6.1, range 66-90). During the 12 -month study period, the median patient filled 10 unique medications (other 2 quartiles: $\mathrm{Q}=7, \mathrm{Q} 3=14$; mean [SD] 11.2 [6.1]) and had 26 pharmacy visits $(\mathrm{Q} 1=18, \mathrm{Q} 3=38$; mean [SD] 30.1 [17.9]). This resulted in a mean (SD) and median synchronization of $0.28(0.18)$ and $0.26(\mathrm{Q} 1=0.15, \mathrm{Q} 3=0.39)$, respectively.

Figure 1 depicts the unadjusted relationship between refill synchronization and polynomial terms for number of medications, including squared, cubed, and fourth and fifth terms. 
TABLE 1 Cohort Characteristics, Pharmacy Experience, and the Association of Patient Characteristics with Synchronization

\begin{tabular}{|c|c|c|c|c|c|c|c|}
\hline \multirow[b]{2}{*}{ Parameter } & \multicolumn{4}{|c|}{ All Medications } & \multicolumn{3}{|c|}{ Chronic Medications $^{\mathrm{a}}$} \\
\hline & $\begin{array}{c}\text { Cohort Distribution } \\
\mathrm{N}=5,248(\%)\end{array}$ & $\begin{array}{l}\text { Parameter } \\
\text { Estimate }\end{array}$ & $\begin{array}{l}\text { Standard } \\
\text { Error }\end{array}$ & $P$ Value & $\begin{array}{l}\text { Parameter } \\
\text { Estimate }\end{array}$ & $\begin{array}{l}\text { Standard } \\
\text { Error }\end{array}$ & $P$ Value \\
\hline Intercept & & 0.260 & 0.0064 & $<0.0001$ & 0.319 & 0.0079 & $<0.0001$ \\
\hline \multicolumn{8}{|l|}{ Number of medications (power) } \\
\hline Number of drugs ${ }^{1}$ & & 0.012 & 9.1E-04 & $<0.0001$ & 0.013 & 0.0012 & $<0.0001$ \\
\hline Number of drugs ${ }^{2}$ & & -0.001 & $9.6 \mathrm{E}-05$ & $<0.0001$ & -0.001 & $2.2 \mathrm{E}-04$ & $<0.0001$ \\
\hline Number of drugs 3 & & $8.2 \mathrm{E}-05$ & $1.6 \mathrm{E}-05$ & $<0.0001$ & $1.4 \mathrm{E}-04$ & $2.3 \mathrm{E}-05$ & $<0.0001$ \\
\hline Number of drugs ${ }^{4}$ & & $2.7 \mathrm{E}-06$ & $1.1 \mathrm{E}-06$ & 0.009 & $-5.0 \mathrm{E}-06$ & $-2.1 E-06$ & 0.017 \\
\hline Number of drugs 5 & & $3.1 \mathrm{E}-08$ & $2.0 \mathrm{E}-08$ & 0.086 & $\mathrm{~b}$ & $\mathrm{~b}$ & $\mathrm{~b}$ \\
\hline \multicolumn{8}{|l|}{ Age, years } \\
\hline $66-70$ & $1,731 \quad(33.0)$ & - & - & - & & & \\
\hline $71-75$ & $1,457 \quad(27.8)$ & -0.0052 & 0.0054 & 0.33 & -0.0051 & 0.0058 & 0.38 \\
\hline $76-80$ & $1,107 \quad(21.1)$ & -0.0083 & 0.0059 & 0.16 & -0.0091 & 0.0064 & 0.15 \\
\hline $81-85$ & $648 \quad(12.3)$ & -0.0078 & 0.0071 & 0.27 & -0.0072 & 0.0077 & 0.35 \\
\hline $86-90$ & $305 \quad(5.8)$ & -0.0056 & 0.0097 & 0.56 & -0.0073 & 0.0104 & 0.49 \\
\hline \multicolumn{8}{|l|}{ Race } \\
\hline White & $\begin{array}{ll}4,588 & (87.4)\end{array}$ & - & - & - & & & \\
\hline African American/black & $394 \quad(7.5)$ & 0.016 & 0.0082 & 0.053 & 0.0057 & 0.0088 & 0.52 \\
\hline Other & $266 \quad(5.1)$ & 0.029 & 0.0097 & 0.0031 & 0.021 & 0.0105 & 0.049 \\
\hline \multicolumn{8}{|l|}{ Ethnicity } \\
\hline Not Hispanic/Latino & $4,932 \quad(94.0)$ & - & - & - & & & \\
\hline Hispanic/Latino & $316 \quad(6.0)$ & 0.0023 & 0.0091 & 0.80 & -0.0025 & .00976 & 0.80 \\
\hline \multicolumn{8}{|l|}{ Comorbidity score } \\
\hline 0 & $2,551 \quad(48.6)$ & - & - & - & - & - & - \\
\hline $1+$ & $2,697 \quad(51.4)$ & 0.026 & 0.0046 & $<0.0001$ & 0.0058 & 0.0051 & 0.25 \\
\hline \multicolumn{8}{|l|}{ Urban/rural } \\
\hline Metropolitan area $>1,000,000$ & $2,870 \quad(54.7)$ & - & - & - & - & - & - \\
\hline Metropolitan area $<1,000,000$ & $1,483 \quad(28.3)$ & 0.015 & 0.0048 & 0.0025 & 0.0164 & 0.0052 & 0.002 \\
\hline Nonmetropolitan $>20,000$ & $302 \quad(5.8)$ & 0.031 & 0.0091 & 0.0006 & 0.032 & 0.010 & 0.001 \\
\hline Nonmetropolitan $<20,000 /$ rural & $593(11.3)$ & 0.082 & 0.0069 & $<0.0001$ & 0.085 & 0.0075 & $<0.001$ \\
\hline \multicolumn{8}{|l|}{ Medicare Part D low-income subsidy } \\
\hline No & $4,112 \quad(78.4)$ & - & - & - & - & - & - \\
\hline Yes & $1,136 \quad(21.6)$ & 0.077 & 0.0057 & $<0.0001$ & 0.067 & 0.0061 & $<0.0001$ \\
\hline \multicolumn{8}{|l|}{ Chemotherapy (history) } \\
\hline No & c & - & - & - & - & - & - \\
\hline Yes & $877 \quad(16.7)$ & -0.0068 & 0.0057 & 0.2303 & -0.0026 & 0.0062 & 0.67 \\
\hline Unknown & $\mathrm{c}$ & c & c & NS & c & c & NS \\
\hline \multicolumn{8}{|l|}{ Radiation therapy (history) } \\
\hline No & $2,011 \quad(38.3)$ & - & - & - & - & - & - \\
\hline Yes & $3,139 \quad(59.8)$ & -0.019 & 0.0045 & $<0.0001$ & -0.0135 & 0.0049 & 0.006 \\
\hline \multirow{2}{*}{ Unknown } & $98 \quad(1.9)$ & 0.027 & 0.016 & 0.087 & 0.028 & 0.017 & 0.11 \\
\hline & Mean (SD) & & & & & & \\
\hline Number of unique medications & $11.19(6.08)$ & & & & & & \\
\hline Number of prescription fills & $46.94(35.10)$ & & & & & & \\
\hline Number of pharmacy visits & $30.09(17.91)$ & & & & & & \\
\hline
\end{tabular}

a Maintenance medications are those intended for long-term use.

bIn this model, since number of medications to the fourth power was not significant, fifth power was not tested.

${ }^{c}$ Due to small cell sizes, the number and results for subjects who did not receive chemotherapy or for whom chemotherapy status was unknown is not reported; model results were adjusted for those variables.

NS = not significant; SD= standard deviation

In a linear regression model (Table 1), the polynomial up to the fourth term of number of medicines remained statistically significantly associated with synchronization, despite adjustments for patient sociodemographic, economic, and health characteristics. Patient comorbidities (other than breast cancer), residence in less metropolitan areas, and receipt of a low-income subsidy for prescription drugs were also associated with higher synchronization. Results were similar in an analysis of only patients who had at least 2 maintenance medications $(n=5,126$; Table 1). 

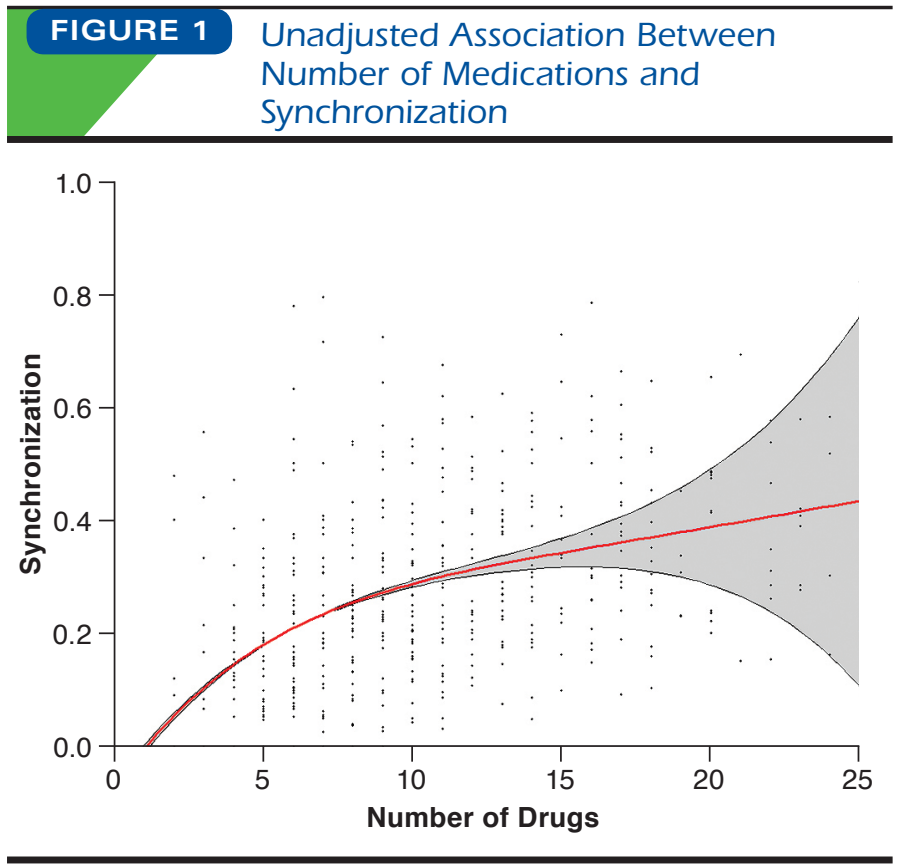

To put our results into perspective, we used parameter estimates from our multivariate models to examine how adjusted synchronization varies with number of medications, along with 2 other characteristics that had the largest effects in the regression model. Results from these analyses showed that with 2 medications, model-predicted synchronization (95\% confidence interval $[\mathrm{CI}])$ was $0.08(0.05-0.10)$, but this increased at 3 medications to $0.13(0.11-0.15)$, for an absolute increase of $4.6 \%$ and a relative increase of $58 \%$. For 7 medications, model-predicted synchronization (95\% CI) was 0.25 (0.23-0.26), and an increase to 8 medications resulted in a more modest predicted increase in synchronization to $0.26(0.25-0.28)$. At 20 medications, the predicted synchronization (95\% CI) was 0.36 (0.34-0.38), which increased for 21 medications to 0.37 (0.35-0.39). Synchronization was also $31 \%$ higher with a prescription medication insurance subsidy, increasing from 0.26 (0.25-0.28) to $0.34(0.32-0.36)$, and $31 \%$ higher in the least urban compared with big metropolitan areas $(0.27[0.25-0.28]$ to 0.35 [0.33-0.37]). For each of these variables, the difference represents the independent effect of the variable on synchronization, after controlling for other potential confounders described in Table 1.

\section{Discussion}

Synchronization of refills has been associated with greater adherence to medications in several studies. Despite this welldocumented association, there has been no clear consensus regarding how best to measure synchronization or how it is associated with the number of medications. Our study contributes to the literature by showing the presence of poor synchronization among a cancer population using a commonly cited measure. More importantly, we also showed that the relationship of synchronization using that measure with number of medications is complex, with a rapid initial rise and a slower, more linear increase after 10 medications. Finally, our study showed that while sociodemographic characteristics, including age and race, were not associated with synchronization, residence in a more rural location and insurance subsidies were associated with higher synchronization.

Our finding regarding the nonlinear relationship of the number of medications with a commonly cited measure of synchronization has potential importance to researchers and policymakers. Although several studies have shown that synchronization programs improve adherence, further research that uses consistent and well-understood measures to examine the mechanisms of the improvements is needed. For example, it is possible that the extensive engagement with pharmacists that is part of many of the synchronization programs explains at least part of the adherence effects. ${ }^{6}$ Reports that describe medication synchronization among users of these programs could be performed, and these reports should account for the subjects' number of medications.

Our results regarding number of medications and synchronization could also be important to support the effectiveness and cost-effectiveness of efforts to implement synchronization programs more widely. Upfront costs of synchronization program can be high, and other forces, such as preferred or specialty pharmacies, may limit the effectiveness of synchronization programs and their dissemination going forward. Furthermore, although pharmacies have been at the forefront of efforts to improve synchronization, there is also growing interest in prescriber-led initiatives, so information such as ours about how to improve measurement of synchronization could foster comparison of approaches. ${ }^{16}$ Synchronization measurement that accounts for its complex relationship with number of medications could allow comparison of the effectiveness and cost-effectiveness of varying adherence-improvement efforts.

We found that urban residence and lack of any prescription insurance subsidy were associated with worse synchronization. Although we could not examine the reasons for the association, rural patients and/or their pharmacies may use systems that allow them to reduce long drives to pharmacies. This finding may also reflect some of the effects of early synchronization programs, since rural pharmacies have led in the development of such programs. ${ }^{4}$ The synchronization in urban areas may also need further attention in policy development and other attempts to help patients in all settings achieve the best possible outcomes. It is possible that high out-of-pocket costs may lead unsubsidized patients to space out their medication refills during the month and that prescription subsidies are associated with better synchronization because they can protect patients from this practice. These hypotheses may be amenable to health policy solutions and should be further examined in other cohorts. 


\section{Limitations}

Our study has some limitations to consider. We focused on patients with a newly started oral cancer therapy for incident early stage breast cancer. Our results may not be generalizable to patients with other chronic conditions. Furthermore, our ability to fully adjust for the severity of patient conditions, which could affect synchronization, was limited to information from the cancer registry and billing data. In addition, we did not have information about pharmacy type (e.g., mail order vs. retail). However, only $5.6 \%$ of Medicare patients used mail-order pharmacies as their primary pharmacies during our study period, ${ }^{17}$ and synchronization has been shown to be associated with adherence in mail-order as well as community pharmacy users. ${ }^{2}$ Nonetheless, it is possible that synchronization will become less important as mail-order use continues to grow.

Finally, our study focused on the Choudhry synchronization index, an intuitive and well-studied measure. Several studies did not provide enough detail regarding their synchronization measures to allow us to assess those measures. We evaluated the possible use of a well-described matrix that used as its primary measure the synchronization of all monthly refills on a single day, but $<5 \%$ of our cohort would have been synchronized by this measure. It is possible, however, that the relationship between synchronization and number of medications may differ if synchronization were to be measured by other metrics.

\section{Conclusions}

Our study uncovered a complex relationship between refill synchronization and number of prescribed medications. Future research into synchronization and adherence should account for this relationship.

\section{Authors}

JOAN M. NEUNER, MD, MPH, and LILIANA PEZZIN, PhD, Department of Medicine and Center for Patient Care and Outcomes Research, Medical College of Wisconsin, Milwaukee. NICOLE FERGESTROM, MS, Center for Patient Care and Outcomes Research, and PURUSHOTTAM W. LAUD, PhD, Division of Biostatistics, Institute for Health and Society and Center for Patient Care and Outcomes Research, Medical College of Wisconsin, Milwaukee.

AUTHOR CORRESPONDENCE: Joan Neuner, MD, MPH, Medical College of Wisconsin, Center for Patient Care and Outcomes Research (PCOR), 8701 Watertown Plank Rd., Milwaukee, WI 53226. Tel.: 414.955.8856; E-mail: jneuner@mcw.edu.

\section{DISCLOSURES}

This study was supported by the National Institute on Minority Health and Health Disparities under grant R01 MD010728. The authors have nothing to disclose.

This study was presented as an oral abstract at the Society of General Internal Medicine Meeting; April 13, 2018; Denver, CO.

\section{ACKNOWLEDGMENTS}

The authors gratefully acknowledge the support of the National Institute on Minority Health and Health Disparities. This study used the linked SEER-Medicare database. The interpretation and reporting of these data are the sole responsibility of the authors. The authors acknowledge the efforts of the National Cancer Institute; the Office of Research, Development and Information, CMS; Information Management Services (IMS); and the Surveillance, Epidemiology, and End Results (SEER) Program tumor registries in the creation of the SEER-Medicare database.

\section{REFERENCES}

1. Choudhry NK, Fischer MA, Avorn J, et al. The implications of therapeutic complexity on adherence to cardiovascular medications. Arch Intern Med. 2011;171(9):814-22

2. Doshi JA, Lim R, Li P, Young PP, Lawnicki VF. Synchronized prescription refills and medication adherence: a retrospective claims analysis. Am J Manag Care. 2017;23(2):98-104.

3. Girdish C, Shrank W, Freytag S, et al. The impact of a retail prescription synchronization program on medication adherence. J Am Pharm Assoc (2003). 2017;57(5):579-84.el

4. Holdford DA, Inocencio TJ. Adherence and persistence associated with an appointment-based medication synchronization program. J Am Pharm Assoc (2003). 2013;53(6):576-83.

5. Holdford D, Saxena K. Impact of appointment-based medication synchronization on existing users of chronic medications. J Manag Care Spec Pharm. 2015;21(8):662-69. Available at: https://www.jmcp.org/doi/10.18553/ jmcp.2015.21.8.662.

6. Krumme AA, Isaman DL, Stolpe SF, Dougherty S, Choudhry NK.

Prevalence, effectiveness, and characteristics of pharmacy-based medication synchronization programs. Am J Manag Care. 2016;22(3):179-86.

7. Warren JL, Klabunde CN, Schrag D, Bach PB, Riley GF. Overview of the SEER-Medicare data: content, research applications, and generalizability to the United States elderly population. Med Care. 2002;40(8 Suppl):IV-3-18.

8. National Cancer Institute. Documentation for the Patient Entitlement and Diagnosis Summary File (PEDSF). November 9, 2016. Available at: https:// healthcaredelivery.cancer.gov/seermedicare/medicare/PEDSF.5percent.pdf. Accessed April 30, 2019.

9. National Comprehensive Cancer Network. NCCN Clinical Practice Guidelines in Oncology (NCCN Guidelines): Breast Cancer. 2013. Available at: https://www.nccn.org/professionals/physician_gls/default.aspx. Accessed April 30, 2019.

10. Klabunde CN, Legler JM, Warren JL, Baldwin LM, Schrag D. A refined comorbidity measurement algorithm for claims-based studies of breast, prostate, colorectal, and lung cancer patients. Ann Epidemiol. 2007;17(8):584-90. 11. Klabunde CN, Potosky AL, Legler JM, Warren JL. Development of a comorbidity index using physician claims data. J Clin Epidemiol. 2000;53(12):1258-67. 12. Deyo RA, Cherkin DC, Ciol MA. Adapting a clinical comorbidity index for use with ICD-9-CM administrative databases. J Clin Epidemiol. 1992:45(6):613-19.

13. Charlson ME, Pompei P, Ales KL, MacKenzie CR. A new method of classifying prognostic comorbidity in longitudinal studies: development and validation. J Chronic Dis. 1987;40(5):373-83.

14. Giordano SH, Duan Z, Kuo YF, Hortobagyi GN, Goodwin JS. Use and outcomes of adjuvant chemotherapy in older women with breast cancer. J Clin Oncol. 2006;24(18):2750-56.

15. Freedman RA, Vaz-Luis I, Barry WT, et al. Patterns of chemotherapy, toxicity, and short-term outcomes for older women receiving adjuvant trastuzumab-based therapy. Breast Cancer Res Treat. 2014;145(2):491-501. 16. Sinsky C. Annual prescription renewal: save time and improve medication adherence. AMA STEPS Forward. October 23, 2014. Available at: https://edhub.ama-assn.org/steps-forward/module/2702751. Accessed April 30, 2019.

17. Marcum ZA, Driessen J, Thorpe CT, Gellad WF, Donohue JM. Effect of multiple pharmacy use on medication adherence and drug-drug interactions in older adults with Medicare Part D. J Am Geriatr Soc. 2014;62(2):244-52. 